\title{
Cell Type Related Differences in Staining with Pentameric Thiophene Derivatives
}

\author{
Artur Cieslar-Pobuda, Marcus Bäck, Karin Magnusson, Mayur Vilas Jain, Mehrdad Rafat, \\ Saeid Ghavami, Peter R. Nilsson and Marek Jan Los
}

\section{Linköping University Post Print}

\section{Tweet}

N.B.: When citing this work, cite the original article.

Original Publication:

Artur Cieslar-Pobuda, Marcus Bäck, Karin Magnusson, Mayur Vilas Jain, Mehrdad Rafat, Saeid Ghavami, Peter J. Nilsson and Marek Jan Los, Cell Type Related Differences in Staining with Pentameric Thiophene Derivatives, 2014, Cytometry Part A, (85A), 7, 628-635.

http://dx.doi.org/10.1002/cyto.a.22437

Copyright: Wiley: 12 months

http://eu.wiley.com/WileyCDA/

Postprint available at: Linköping University Electronic Press

http://urn.kb.se/resolve?urn=urn:nbn:se:liu:diva-109171 


\section{Cell type related differences in staining with pentameric thiophene derivatives}

Running title: Fluorescence of pentameric thiophene derivatives

Artur Cieślar-Pobuda ${ }^{1,2 *}$, Marcus Bäck ${ }^{3 *}$, Karin Magnusson ${ }^{3}$, Mayur V. Jain ${ }^{1}$, Mehrdad Rafat $^{1}$, Saeid Ghavami ${ }^{4}$, K. Peter R. Nilsson ${ }^{3 \#}$, and Marek J. Los ${ }^{1,5 \#}$

${ }^{1}$ Department Clinical and Experimental Medicine (IKE), Division of Cell Biology, and Integrative Regenerative Medicine Center (IGEN), Linköping University, Sweden;

${ }^{2}$ Biosystems Group, Institute of Automatic Control, Silesian University of Technology, Gliwice, Poland;

${ }^{3}$ Department of Chemistry, IFM, Linköping University, Sweden;

${ }^{4}$ Department of Human Anatomy and Cell Science, Manitoba Institute of Child Health, Department of Physiology, St. Boniface Research Centre, University of Manitoba, Canada,

${ }^{5}$ Department of Pathology, Pomeranian Medical University, Szczecin, Poland.

* Correspondence address:

Marek Łos, MD/PhD,

Dept. Clinical and Experimental Medicine (IKE)

Integrative Regenerative Medicine Center (IGEN)

Linköping University

Cell Biology Building, Level 10

58185 Linköping, Sweden

Email: marek.los@liu.se, T: +46-10-10 32787

\section{Keywords:}

cancer stem cells, luminescent conjugated oligothiophenes, fluorescent probes,

List of abbreviations: AD- Alzheimer's disease; CSCs- Cancer Stem Cells; DAPI- 4',6Diamidino-2-Phenylindole; FBS- Fetal Bovine Serum; LCOs- Luminescent Conjugated Oligothiophenes; LCPs- Luminescent Conjugated Polythiophenes; PBS- Phosphate Buffered Saline; PS- Pencillin Streptomycin; PTAA- Polythiophene Acetic Acid. 


\begin{abstract}
Fluorescent compounds capable of staining cells selectively without affecting their viability are gaining importance in biology and medicine. Recently, a new family of optical dyes, denoted luminescent conjugated oligothiophenes (LCOs), has emerged as an interesting class of highly emissive molecules for studying various biological phenomena. Properly functionalized LCOs have been utilized for selective identification of disease-associated protein aggregates and for selective detection of distinct cells. Herein, we present data on differential staining of various cell types, including cancer cells. The differential staining observed with newly developed pentameric LCOs is attributed to distinct side chain functionalities along the thiophene backbone. Employing flow cytometry and fluorescence microscopy we examined a library of LCOs for stainability of a variety of cell lines. Among tested dyes we found promising candidates that showed strong or moderate capability to stain cells to different extent, depending on target cells. Hence, LCOs with diverse imidazole motifs along the thiophene backbone were identified as an interesting class of agents for staining of cancer cells, whereas LCOs with other amino acid side chains along the backbone showed a complete lack of staining for the cells included in the study. Furthermore, for p-HTMI,a LCO functionalized with methylated imidazole moieties, the staining was dependent on the p53 status of the cells, indicating that the molecular target for the dye is a cellular component regulated by $\mathrm{p} 53$. We foresee that functionalized LCOs will serve as a new class of optical ligands for fluorescent classification of cells and expand the toolbox of reagents for fluorescent live imaging of different cells.
\end{abstract}




\section{INTRODUCTION}

The ability of selective identification of biomolecules in various cells is undoubtedly of great importance. No less crucial is the use of proper probes that are characterized with high fluorescence and high photostability that allow detection of low-abundance biological structures with great sensitivity and selectivity. Most of these properties have been achieved by the development of luminescent conjugated oligothiophenes (LCOs). Oligothiophene molecules are well known mainly for their semi-conductive properties and their usefulness in photonics and organic electronics (1). However these compounds have also been applied in fluorescent imaging of biological structures (2-9). The fact that they are generally nontoxic (2) additionally makes them even more suitable for cellular staining and real-time imaging with fluorescence microscopy. It has been shown that oligothiophene dyes can be successfully used in direct covalent labeling of distinct biomolecules, such as nucleosides and oligonucleosides, as well as proteins $(3,4)$.

Besides covalent labeling of biomolecules, functionalized LCOs can also be applied as selective and specific ligands towards distinct biomolecular targets, such as protein aggregates or specific cells (5-15). LCOs allow for identification of protein aggregates associated with various degenerative diseases, such as Alzheimer's disease (AD) or prion diseases, in a wide range of experimental models, as well as in vivo (5-9). When LCOs interact with disease associated protein aggregates, the conformational freedom of the flexible thiophene backbone is restricted and the emission properties of the dyes are affected in a conformation-dependent manner (11). Compared to other conventional ligands, LCOs has proven useful for a wider range of disease-associated heterogeneous protein aggregates, including protein inclusion bodies (12-15).

Luminescent conjugated polythiophenes (LCPs) have also been utilized for staining of cells. Lysosome-related acidic vacuoles were targeted in cultured primary cells by anionic, cationic, and zwitterionic polythiophene derivatives, whereas the targeting of lysosome-related acidic vesicles could not be demonstrated in malignant cells (9). The anionic LCP, polythiophene acetic acid (PTAA), could also be used for vital staining of live cells. Recently, p-HTMI, a pentameric LCO functionalized with a methylated imidazole moiety, was identified as a potential marker for neural stem cells and glioma derived stem cells, whereas nonmethylated imidazole analogues were not functional, suggesting a crucial chemical side chain functionalization for the specificity of the molecule. Hence, LCOs with distinct side chain functionalities may provide a novel generation of molecular probes for immediate and specific detection of various cell types.

Herein, we screen a library of pentameric LCOs (Fig. 1A) towards various cell types, mostly cancer cells, with the aim of evaluating LCO staining propensity of cells by flow cytometry and fluorescent microscopy. The objective was to examine if various functionalized LCOs can be used for specific staining of distinct cell subpopulations, since fluorescent dyes that are able to distinguish for example cancer stem cells (CSCs) from a heterogenous population of cancer cells would bring a tremendous impact on further investigations in the field of stem cell biology. We found promising candidates that showed strong or moderate capability to stain cells to different extents, depending on the cell line. LCOs having diverse imidazole functionalizations along the thiophene backbone were identified as an interesting class of agents for cell staining of cancer-associated cells and for p-HTMI, a pentameric LCOs functionalized with methylated imidazole moieties, the staining was dependent on the p53 status of the cells 
(O. Hermansson, pers. communication). We foresee that properly functionalized LCOs will serve as new optical reagents for fluorescent classification of cancer-associated cells.

\section{MATERIALS AND METHODS}

\section{Synthesis and Optical Characterization of Luminescent Conjugated Oligothiophenes}

p-HTAA, p-HTMI, and p-HTIm were synthesized as previously described (5). p-HTEA, pHTA-Histamine, p-HTA-Tyr, p-HTA-Arg, and p-HTA-Glu were synthesized in a similar fashion and the detailed synthesis and synthetic schemes of these dyes are provided in the supplementary material. All of the dyes were dissolved in deionized water to a final concentration of $1.5 \mathrm{mM}$, and further diluted to a final concentration of $1.5 \mu \mathrm{M}$ with PBS. Emission spectra were collected between 450-650 nm using a Tecan Saphire 2 plate reader with excitation at $420 \mathrm{~nm}$ after $10 \mathrm{~min}$ of incubation. Similarly, excitation spectra between 300 and $500 \mathrm{~nm}$ were measured for all of the samples. 

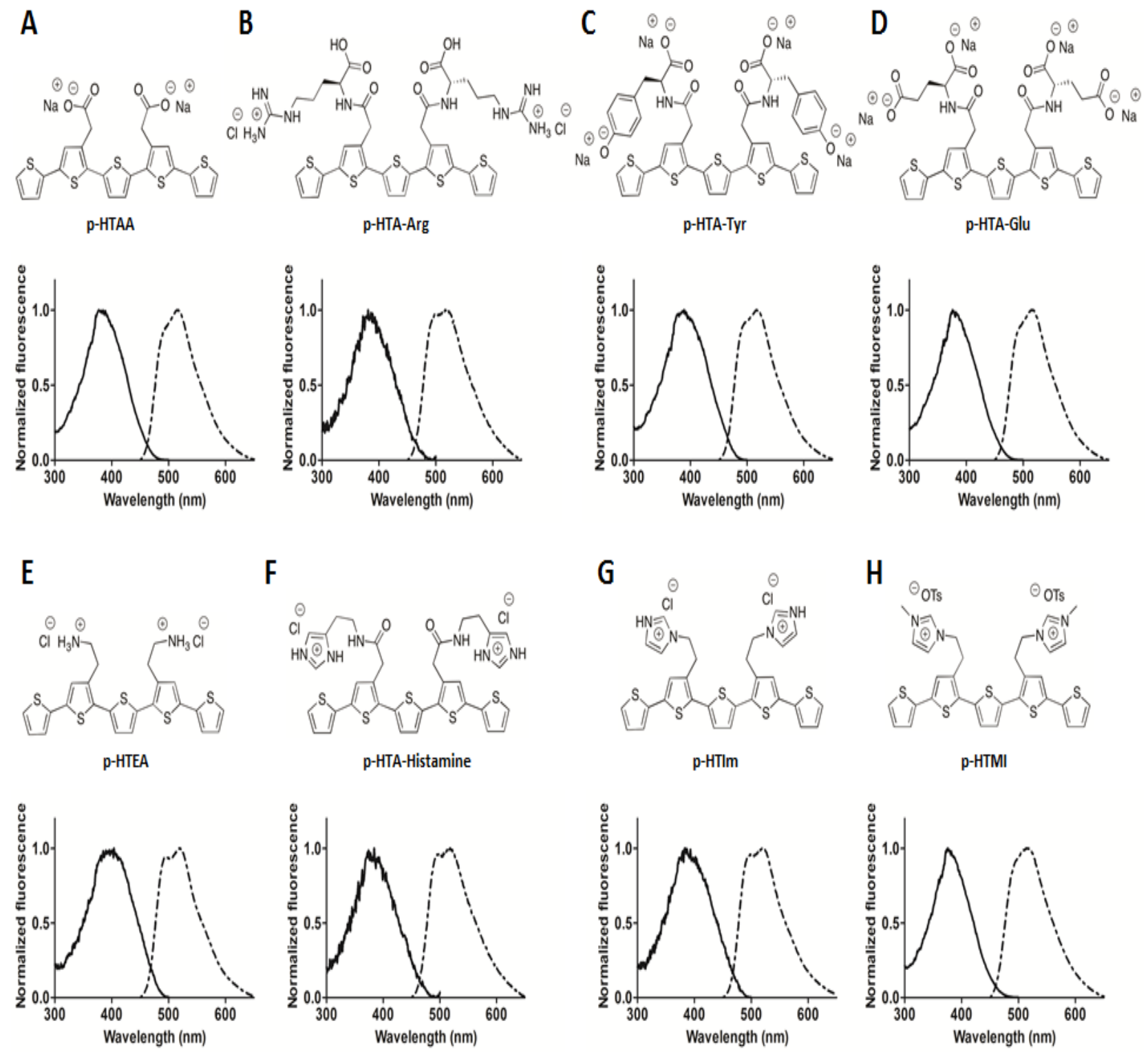

Figure 1. Chemical structures and excitation- linesand emission spectrum (dotted lines) of p-HTAA (A), p-HTA$\operatorname{Arg}(B)$, p-HTA-Tyr (C), p-HTA-Glu (D), p-HTEA (E), p-HTA-Histamine (F), p-HTIm (G) and p-HTMI (H). The spectra were recorded for $1.5 \mu \mathrm{M}$ of the respective LCO in PBS pH 7.4.

\section{Cell Lines and Cell Culture}

Experiments were performed on following human cell lines: SKBR-3, MDA-MB-468, MCF-7 (breast cancer cell lines), PC-3, DU-145 (prostate cancer cell lines), HeLa (cervical cancer), HCT 116 (colon cancer cell line; HCT 116 wt and HCT 116 p53 $3^{-/}$), K562 (chronic myelogenous leukemia cells), HEK-293 (human embryonic kidney cells) and primary human dermal fibroblasts. Cells were cultured either in RPMI-1640 medium (PAA, Austria) (SKBR-3, MDA-MB-468, K562) or in DMEM High Glucose (PAA, Austria) supplemented with $10 \% \mathrm{v} / \mathrm{v}$ inactivated fetal bovine serum (FBS) (PAA, Austria) and 1\% v/v pencillin streptomycin (PS) (Gibco, USA) and maintained in $37^{\circ} \mathrm{C}$ in humidified with $5 \%$ carbon dioxide. After reaching 
$70 \%$ to $80 \%$ confluency cells were trypsinised by Trypsin EDTA 1x (PAA, Austria) (or just collected as in a case of K562 cells growing in suspension) and exposed for oligothiophene staining.

\section{Staining of Cells}

Dyes were added to cells in culture medium at final concentration $1.5 \mu \mathrm{M}$. After $0.5 \mathrm{~h}$ of incubation at $37^{\circ} \mathrm{C}$ in dark, cells were washed twice with PBS, re-suspended in fresh culture medium and analyzed using flow cytometry.

\section{Flow Cytometric Analysis}

Fluorescence was measured by a Gallios flow cytometer (Beckman Coulter) using FL1 channel. The number of events was stopped at 20,000 counts. Data collected from the experiments were analyzed using Kaluza (analysis software from Beckman Coulter).

\section{Microscopic Analysis}

Cells were seeded at a density of $5 \times 10^{4}$ on coverslips in 6-well plates and allowed to attach. After overnight incubation, medium was changed with fresh one and supplemented with $1.5 \mu \mathrm{M}$ oligothiophene dyes. After $0.5 \mathrm{~h}$ of staining at $37{ }^{\circ} \mathrm{C}$, cells were washed twice with PBS, fixed with $4 \%$ paraformaldehyde for 20 minutes at room temperature and again washed with PBS. To visualize nuclei localization cells were co-stained with DAPI $(1 \mu \mathrm{g} / \mathrm{ml})$. Images were taken using confocal microscope (Zeiss). Alternatively, for live staining, dyes at the same concentrations were added to cells in culture and after approximately 30 minutes pictures were taken by $\mathrm{JuLI}^{\mathrm{TM}}$ fluorescent cell analyzer $(10 \times$ magnification $)$.

\section{RESULTS}

\section{Synthesis and Optical Characterization of Luminescent Conjugated Oligothiophenes}

To achieve a library of LCOs, we designed a class of pentameric LCOs with distinct side chain functionalities and all LCOs (Fig. 1) were synthesized using previously reported trimeric and pentameric building blocks (5) (Schemes 1 and 2, supplementary information). The anionic LCO, p-HTAA (Fig. 1A), has two carboxyl moieties along the thiophene backbone, whereas pHTEA (Fig. 1E) is functionalized with amine groups rendering a cationic LCO under physiological conditions. p-HTAA was further functionalized with four amino acid derivatives to achieve, p-HTA-Glu (Fig. 1D), p-HTA-Arg (Fig. 1B, p-HTA-Tyr (Fig. 1C) and p-HTAHistamine (Fig. 1F). Furthermore, two additional LCOs, p-HTIm (Fig. 1G) and p-HTMI (Fig. $1 \mathrm{H})$, having imidazole or methylated imidazole side chain functionalities were included in the library. In PBS pH 7.4, all the LCOs showed excitation and emission maxima around 380-400 $\mathrm{nm}$ and 515-530 nm, respectively (Fig. 1). Hence, all LCOs showed a Stokes shift of approximately $140 \mathrm{~nm}$. The minor spectral variation between the dyes is a consequence of the chemical modifications of the substituents attached to the pentameric thiophene scaffold.

\section{Labeling of Cells by Luminescent Conjugated Oligothiophenes}

Microscopic observations of cells treated with LCOs showed that tested dyes could be successfully used for staining both fixed (Fig. 2A) and live (Fig. 2B) cells. Confocal imaging revealed that LCOs accumulate in cytoplasm and even if cells were previously permeabilized, dyes found their molecular targets only outside the nucleus. In a case of live staining, 
compounds were not toxic to examined cells, as number of viable cells did not change significantly even after 24 hours incubation with LCOs ( $>95 \%$ live cells, as measured by a trypan blue staining). Furthermore, the staining was stable on live cells for up to four days. Among eight tested LCOs two of them p-HTEA and p-HTIm, at a concentration of $1.5 \mu \mathrm{M}$, labeled cells with high intensity. These two dyes stained all examined cell lines and the fluorescence was only weaker in a case of fibroblasts. The rest of dyes showed much lower fluorescence level or no stainability at all.

The level of staining in cells was difficult to compare only by microscopy imaging, thus for quantitative analysis flow cytometer was used. It enabled us to monitor the integrated intensity of fluorescence for each cell, get the average intensity for cell populations and compare histograms (since fluorescence distributions provide more information than single values of mean or median)(Fig. 3). In most cases we did not observe any partially stained populations e.g. multiple peaks or distributions with long tails. Histograms mostly comprised only one single peak, which suggests that examined cells uniformly stained with LCOs. Histograms differed however in their width, which tells that among labeled cells, some have higher fluorescence signal intensity than the others. However, for some cell lines (SKBR-3, MCF-7, HCT-116), staining by p-HTMI and p-HTAA showed that a fraction of cells $(\sim 1-2 \%)$ is stained more intensively than the main cell population (Fig. 3B). Such observation was specific

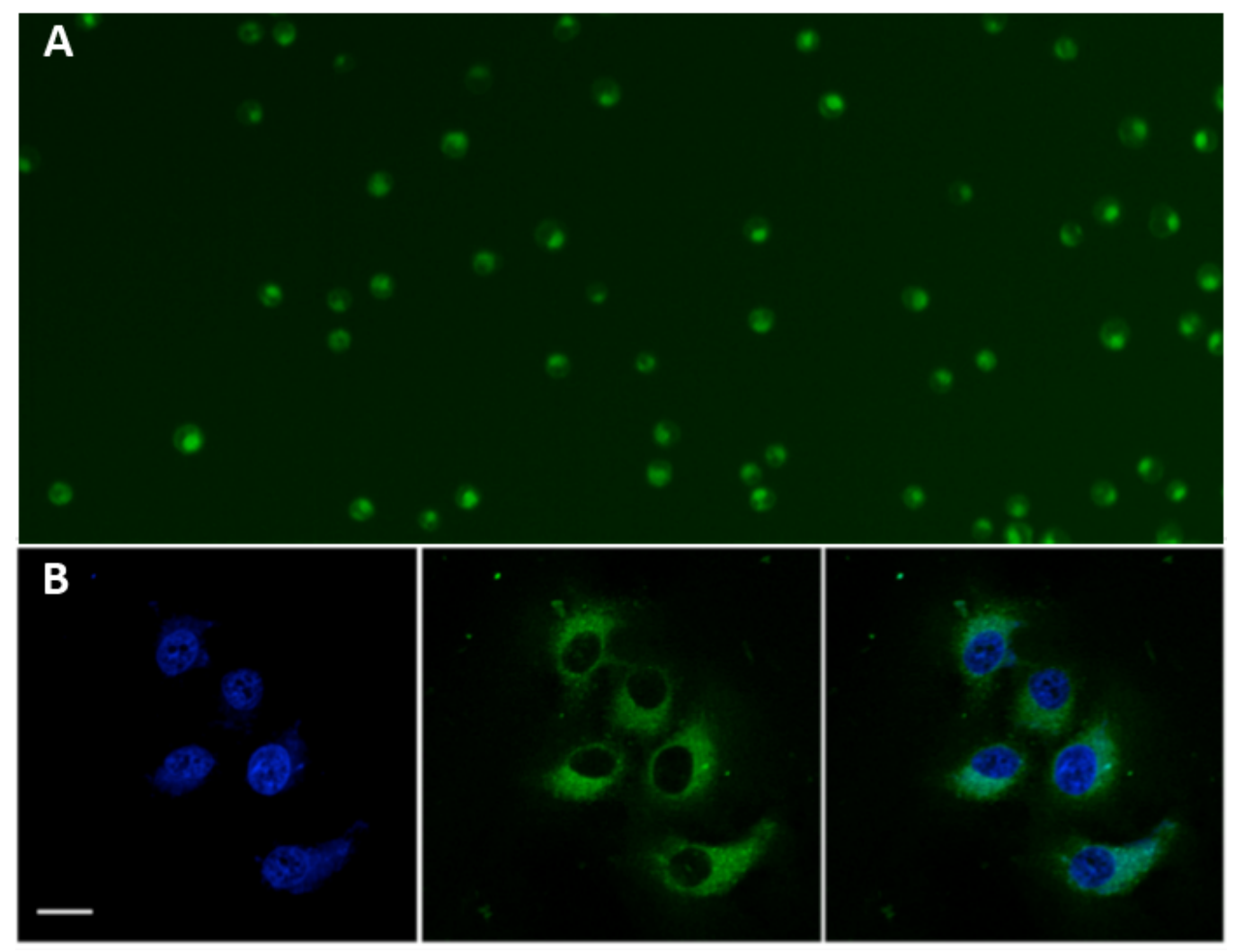

Figure 2. Fluorescence images with p-HTEA upon staining of live and fixed cells. (A) Alive leukemia K562 cells grown in suspension (magnification $\times 10$ ). (B) Fixed prostate cancer PC3 cells stained by p-HTEA (green fluorescence) with nuclei co-stained with DAPI (blue fluorescence). Scale bar represents $20 \mu \mathrm{m}$. 
mainly for breast cancer cells and suggests that more-intensively stained subpopulation may represent cells with different properties (e.g. cancer stem cells). Results showed also that tested LCOs stain various cell lines to different extent (Table 1). Such comparison was done by gating unstained cells and then by applying the same gate (or threshold) to samples treated with LCOs. Percentage of cells over the threshold value of green fluorescence was considered as the measure of stainability.

Detailed analysis of eight dyes and ten cell lines showed that two LCOs, p-HTIm and pHTEA, possess the strongest labeling properties. All cells treated with these two dyes were stained and the green fluorescence had the highest values in a case of p-HTEA $\left(>10^{2}\right.$ fluorescence units $<$ FL1 INT $>$ as revealed by flow cytometry).

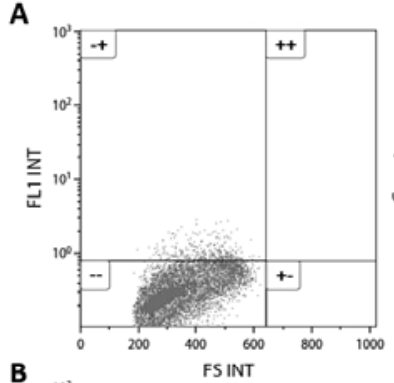

B

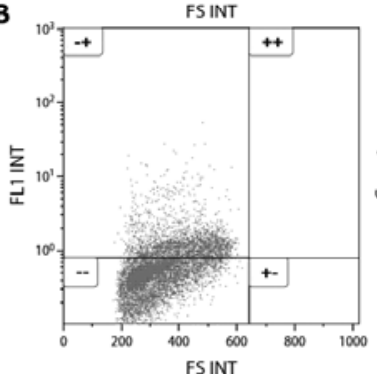

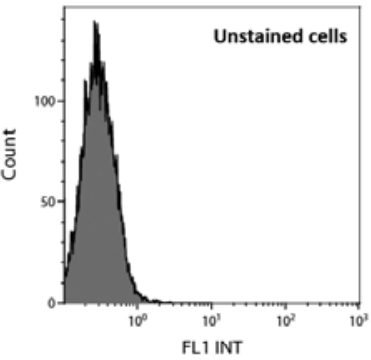

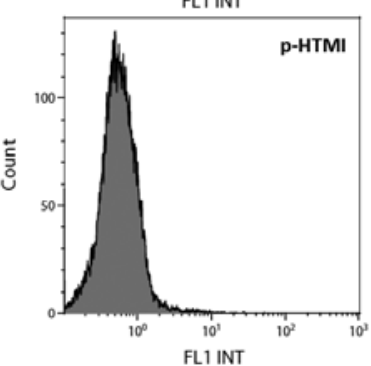

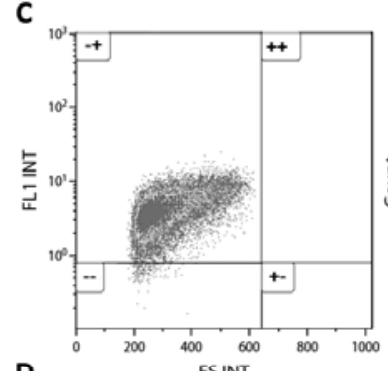
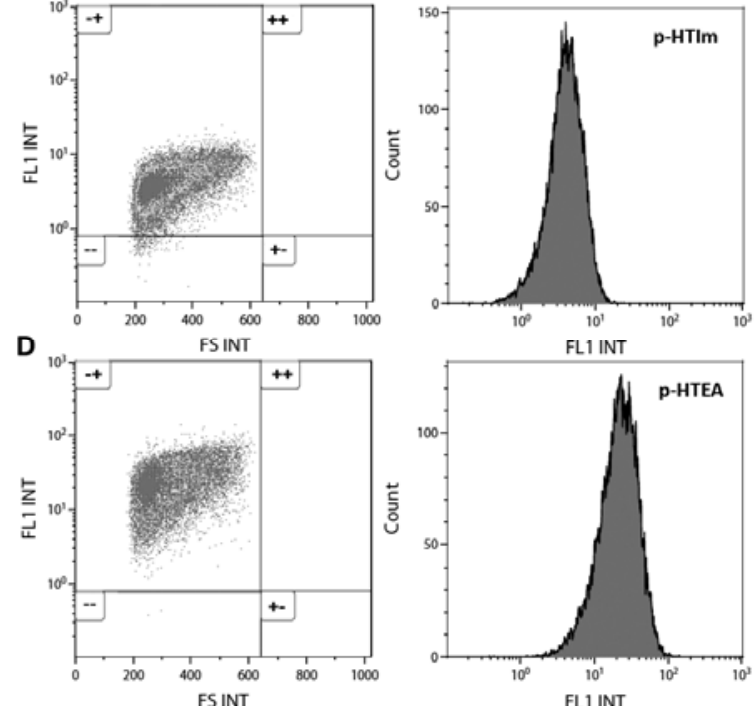

Figure 3. Representative forward scatter (FS INT) vs. fluorescence (FL1 488/525) dot pots and their corresponding histograms of stained colon cancer HCT116 cells (wt). The signals were detected with the $488 \mathrm{~nm}$ laser and 525/40 BP filter.(A) unstained cells, (B) cells stained with p-HTMI (a small group of cells with high fluorescence signal is visible above the main population of cells), (C) cells stained with p-HTIm, (D) cells stained with p-HTEA. The level of stainability was determined on a basis of percentage of cells in "-+" (upper left) quadrant.

LCOs whose pentameric oligothiophene backbone was functionalized with the amino acids, Arg, Tyr, or Glu did not stain cells in a concentration of $1.5 \mu \mathrm{M}$ or $3 \mu \mathrm{M}$, whereas pHTMI, p-HTA-His and p-HTAA stained various cell lines to different extent. Notably, the three LCOs functionalized with distinct imidazole moieties, p-HTMI, p-HTIm and p-HTA-Histamine, stained the cells with different fluorescence intensity, despite their rather similar chemical structures. Hence, small modification of the imidazole side chain, such as adding methyl groups or attaching the imidazole moiety in different fashions (carbon or nitrogen) lead to altered staining properties of the pentameric LCO. Overall, we conclude that some LCOs seem to stain various cell lines at different level (p-HTAA, p-HTMI and p-HTA-histamine) whereas p-HTEA and p-HTIm showed strongest propensity to stain cells.

It is also worthy to notice that the normal cell line used in this study, primary human fibroblasts, showed very week stainability by a majority of tested LCOs. Only p-HTEA labeled fibroblasts on a similar level as other cells. The second strongest dye: p-HTIm stained only 
Table 1. Stainability of different cell lines with oligothiophene derivatives (1.5 $\mu \mathrm{M})$. Numbers refer to percentage of cells stained with tested dyes (as compared to unstained cells and measured by flow cytometry). For clarity only average values (without std. dev.) from 2-4 repeats are presented. Colors represent different levels of staining.

\begin{tabular}{|c|c|c|c|c|c|c|c|c|c|c|c|}
\hline Name & Side chain & $\begin{array}{c}\text { SKBR- } \\
\mathbf{3}\end{array}$ & $\begin{array}{c}\text { MDA- } \\
\text { MB- } \\
\mathbf{4 6 8}\end{array}$ & MCF7 & Hela & HCT116 & DU-145 & HEK & PC-3 & K562 & Fibroblasts \\
\hline p-HTAA & $\begin{array}{c}\text { Anionic } \\
\text { (-COOH) }\end{array}$ & 5,92 & 9,02 & 11,43 & 23,21 & 12,47 & 7,66 & 0,07 & 0,34 & 5,82 & 0,09 \\
\hline p-HTEA & $\begin{array}{c}\text { Cationic } \\
\text { (-NH3) }\end{array}$ & 100,00 & 100,00 & 100,00 & 100,00 & 100,00 & 100,00 & 100,00 & 100,00 & 100,00 & 99,30 \\
\hline p-HTMI & $\begin{array}{c}\text { Methylated } \\
\text { imidazole }\end{array}$ & 21,63 & 72,46 & 27,45 & 26,34 & 11,83 & 64,93 & 79,38 & 74,05 & 29,20 & 0,17 \\
\hline $\begin{array}{c}\text { p-HTA- } \\
\text { Histamine }\end{array}$ & Histamine & 32,67 & 49,30 & 6,94 & 2,40 & 14,54 & 25,45 & 0,77 & 8,30 & 59,64 & 0,00 \\
\hline p-HTIm & Imidazole & 98,00 & 99,00 & 95,96 & 99,00 & 98,18 & 98,21 & 99,90 & 99,14 & 99,40 & 22,24 \\
\hline $\begin{array}{c}\text { p-HTA- } \\
\text { Tyr }\end{array}$ & Tyrosine & 0,23 & 0,25 & 0,00 & 0,28 & 0,24 & 0,12 & 0,05 & 0,08 & 0,00 & 0,14 \\
\hline $\begin{array}{c}\text { p-HTA- } \\
\text { Arg }\end{array}$ & Arginine & 0,36 & 0,00 & 0,00 & 0,07 & 0,00 & 0,00 & 0,00 & 0,06 & 0,00 & 0,09 \\
\hline $\begin{array}{c}\text { p-HTA- } \\
\text { Glu }\end{array}$ & Glutamate & 1,94 & 0,00 & 0,00 & 0,39 & 0,12 & 0,00 & 0,07 & 0,15 & 0,00 & 0,00 \\
\hline
\end{tabular}

$\sim 22 \%$ of fibroblasts. Other LCOs were unable to label fibroblasts within 30 minutes at concentration of $1.5 \mu \mathrm{M}$. Interestingly, decreasing the concentration of p-HTEA and p-HTIm to $0.25 \mu \mathrm{M}$ made fibroblast completely staining-negative, whereas other cells still emitted a fluorescent signal. Hence, at lower concentrations both p-HTEA and p-HTIm showed specific labeling towards the investigated cancer cell lines.

\section{LCOs Staining of Cells with Different p53 Status}

The next step of our studies was to investigate if LCOs' stainability could depend on the expression of some proteins important for the cell's functionality. For this purpose we chose colorectal cancer HCT1-116 cells that differ in the status of p53 expression. Both cell lines were stained by four dyes: p-HTAA, p-HTMI p-HTA-Histamine and p-HTIm. For the latter, working concentration was decreased from $1.5 \mu \mathrm{M}$ to $0.25 \mu \mathrm{M}$ to better visualize the differential staining propensity. As shown in figure 4, more HCT116 WT cells were stained with p-HTAA, p-HTIm and p-HTA-Histamine than HCT116 p53/- cells.

On the other hand, a higher percentage of labeled HCT116 $\mathrm{p} 53^{-/-}$than HCT116 wt cells was observed after staining with p-HTMI. Such a trend was not observed for any other dyes, suggesting that such staining is rather not a result of different condition of both cell types in a culture but that indeed there are some cellular components regulated by p53 that is the molecular target for p-HTMI.

\section{Effect of Prolonged Cells Incubation with LCOs}

After screening of all LCOs we checked whether a longer incubation with dyes might alter the staining characteristics. We limited the number of tested compounds to the ones that showed moderate or non-staining properties. Incubation of cells with compounds: p-HTA-Tyr, p-HTAArg, p-HTA-Glu, for 90 minutes did not result in the appearance of fluorescent signal. In the case of remaining LCOs, longer incubation generally caused higher level of fluorescence in 
cells (Fig. 5). The highest increase of fluorescence was observed in a case of SKBR3 (breast cancer) and K562 (leukemia) cells incubated with p-HTA-Histamine and p-HTMI dyes (Fig. 5 $\mathrm{AB})$. These dyes, after 90 minutes of incubation, stained only $6 \%$ of fibroblasts.

For both K562 and SKBR-3 cells, we observed a similar kinetic of staining by LCOs: fluorescence increased rapidly during first 30-45 minutes and then cells were becoming saturated with dyes. Further incubation with staining agents caused only a small rise in the fluorescence. The exception is p-HTMI, which stained cells with increased intensity in a timedependent manner.

\section{DISCUSSION}

Our study focuses on characterization of a library of pentameric conjugated oligothiophenes towards different cell types. As shown in previous papers, LCOs have successfully stained protein aggregates associated with various degenerative diseases like prion or Alzheimer's disease (5-7) and detected intracellular inclusion bodies (12). Here we have demonstrated that several LCOs (p-HTAA, p-HTEA, p-HTMI, p-HTA-Histamine and p-HTIm) can also be effectively used in cellular staining, making it possible to distinguish some type of cells from others. Our test of LCOs on various cell types revealed new propensities of examined oligothiophene molecules.

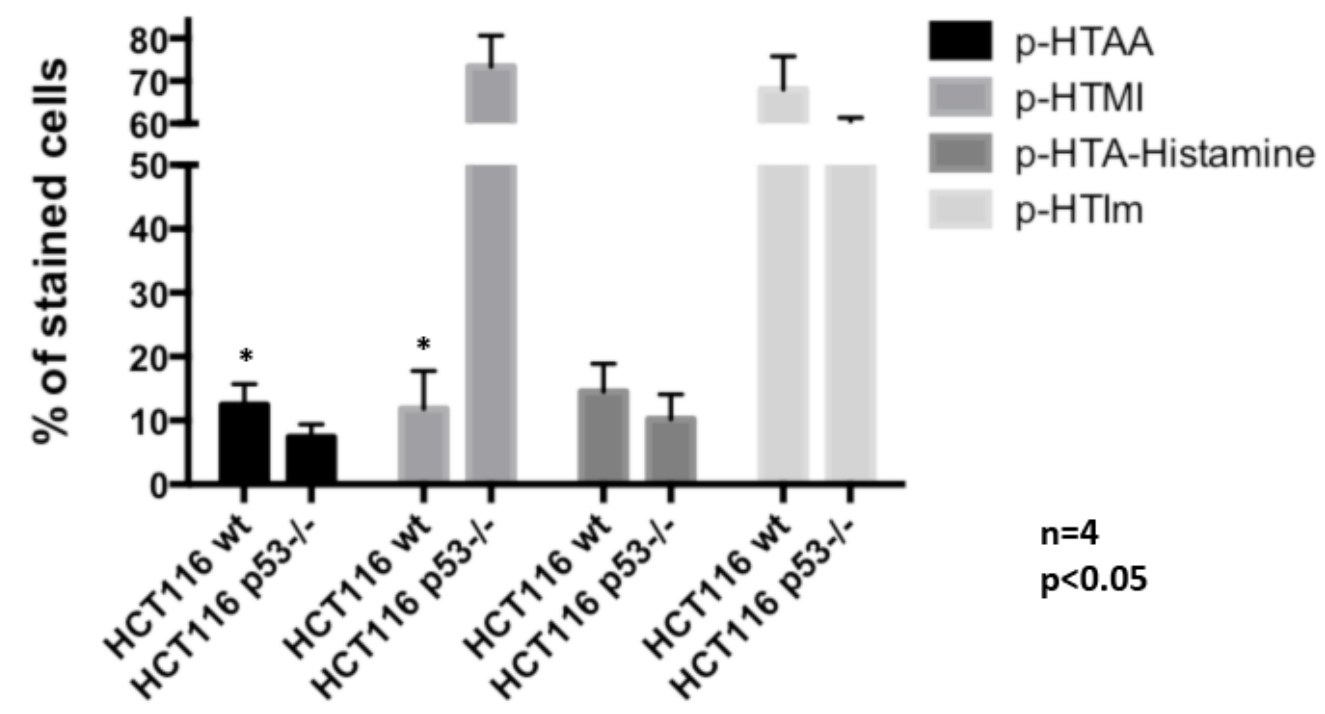

Figure 4. Stainability of HCT116 cells differing in p53 status. Results are expressed as the mean percentage of labeled cells \pm SD. * Denotes significant differences between two cell lines. ( $n=4 ; p<0.05$; Student's t-test) stained with the same LCO. Concentration of p-HTIm used in these studies was decreased 6 times in comparison to other dyes and previous experiments.

Our experiments revealed that some cells stained with individual LCOs stronger than others. It was especially prominently visible in the case of p-HTA-Histamine, which preferentially stained K562 cells (Table 1). On the other hand, also some similarities in staining pattern were observed; especially between cells from the same tumor types. Some LCOs stained the breast cancer lines: SKBR-3, and MDAMB-468 with a similar intensity, suggesting that alike molecular targets for the tested LCOs are present in both cell lines. Likewise, the discrepancy in LCO stainability for various cells line can be a result of different molecular 

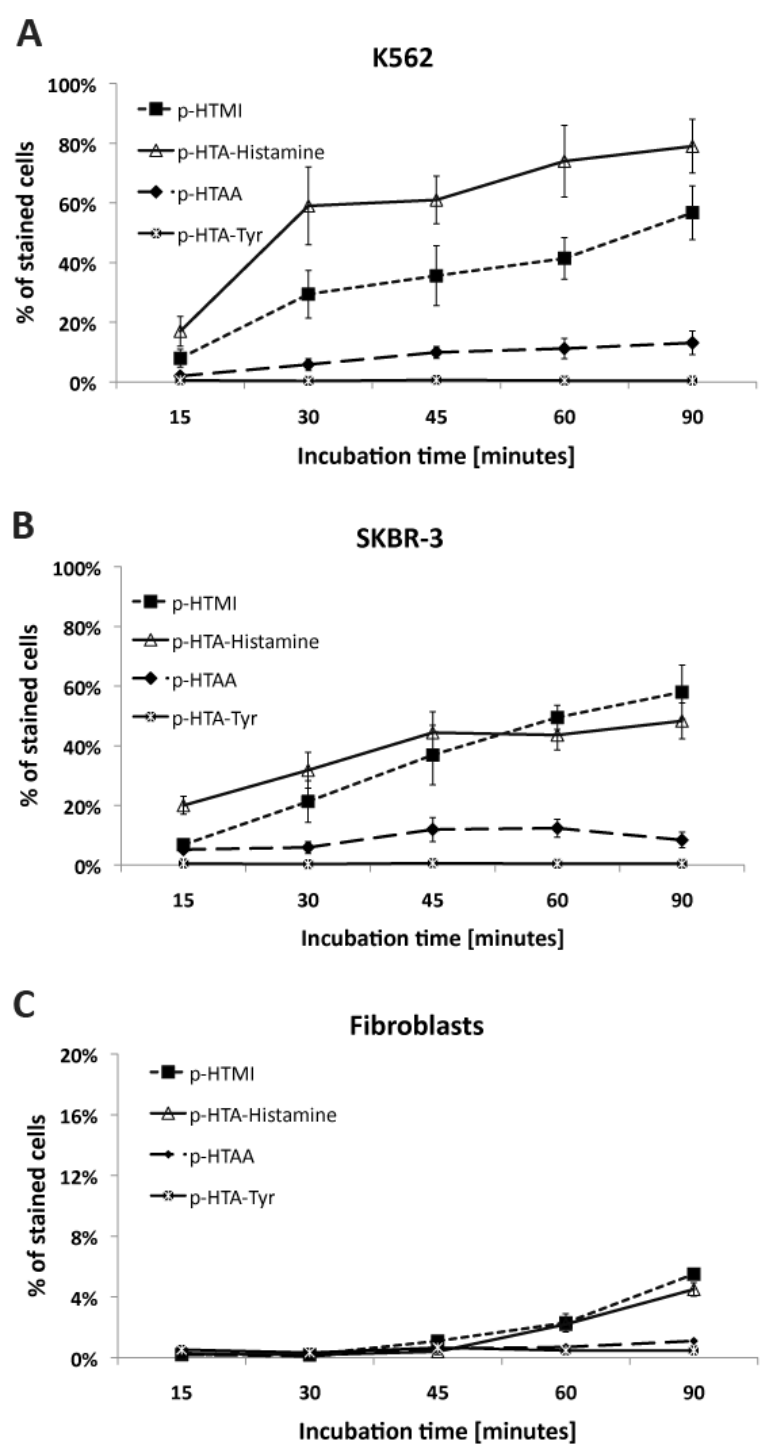

Figure 5. Change of the number of cells stained by LCOs when different incubation times are applied in various cell lines. (A) results for myelogenous leukemia K562 cells; (B) results for breast cancer SKBR-3 cells, (C) results for primary human dermal fibroblasts. (Note that in figure "C" y-scale is up to only $20 \%$ ). Values are presented as mean \pm SD from three independents experiments.

targets or their different amount in examined cell types. LCOs are also known to distinguish conformational changes of peptides and proteins (11) thus it is also probable that LCOs binds with different affinity to the same protein that exists in altered conformation in different cells. As we know, conformation of protein or peptide depends not only on the order of amino acids but is also influenced by numerous factors in the surrounding.

As shown in our studies, compounds: p-HTA-Tyr, p-HTA-Arg, p-HTA-Glu, did not stain examined cells. It means that either these molecules could not enter the cell or they did not bind with sufficiently high affinity to any intracellular structures. It is rather unlikely that the size of LCO is the main obstacle for successful staining, as in previous studies it was demonstrated that cells can be stained by molecules of conjugated oligothiophenes with structures consisting of more than five thiophene rings (12) and also by luminescent conjugated polymers (9) of much higher molecular weights. Overall, our findings suggest that specificity towards distinct cells can be obtained through incorporation of a specific side chain functionalization along the pentameric thiophene backbone. 
Interestingly, we have also noticed that among the examined cell types, primary human dermal fibroblasts were the only cells that were almost completely unstainable by the tested LCOs. Although fibroblasts were stained by p-HTEA and p-HTIm, their fluorescence was considerably weaker than the fluorescence of the other cells stained by these dyes. Decreasing the concentration of p-HTEA and p-HTIm from $1.5 \mu \mathrm{M}$ to $0.25 \mu \mathrm{M}$ made fibroblast fluorescencenegative, whereas other cells still emitted fluorescent signal. Fibroblasts have been difficult to identify, especially in histological materials. Very frequently fibroblasts are identified only on a basis of their morphology (spindle shape) combined with positive staining for the mesenchymal marker vimentin (16). A few antibodies have also been reported to recognize fibroblasts, some of which take advantage of fibroblasts as the major producer of collagen (17). Inability of fibroblasts to be stained by pentameric LCOs is also in contrast to the results from a previous study using anionic and cationic polythiophenes (9). The anionic polythiophene, PTAA, clearly labeled lysosome-related acidic vesicles in cultured primary fibroblasts, whereas these vesicles could not be demonstrated in transformed cells. Taken together, our results and the results from this earlier study (9) indicate that the length of the repetitive thiophene backbone is also a crucial determinant for selective staining of distinct cells. From this perspective, it will be interesting to test the library of pentameric LCOs, as well as LCOs with extended backbone on more types of normal and primary cells.

Table 2. Stainability of cell lines with p-HTEA and p-HTIm at concentration $0.25 \mu \mathrm{M}$. Numbers refer to percentage of cells stained with tested dyes (as compared to unstained cells and measured by flow cytometry).

\begin{tabular}{|c|c|c|c|c|c|c|}
\hline Name & $\begin{array}{c}\text { SKBR- } \\
\mathbf{3}\end{array}$ & HCT116 & MCF7 & PC-3 & HELA & Fibroblasts \\
\hline p-HTEA & 99,1 & 97,16 & 86,8 & 99,72 & 73,2 & 1,09 \\
\hline p-HTIm & 91,24 & 68,2 & 24,3 & 77,55 & 14,73 & 0,21 \\
\hline
\end{tabular}

One of the sought-after properties of new dyes in oncology is their ability to detect cancer stem cells (CSCs). The cancer stem cells concept states that cancer cells may include a subgroup of cancer propagating cells that have the ability of self-renewal and are capable of generating the diverse tumor cells (18). CSCs have been isolated from several types of cancers, including but not limited to breast-, brain-, skin-, head \& neck-, thyroid-, cervix-, retina-, lung cancer, and from leukemias and lymphomas (19-22). Surely, the efforts aimed at efficient targeting of CSCs by LCOs would be important for monitoring the progress of cancer therapy and for evaluating new therapeutic approaches. Our studies did not bring an explicit answer whether or not tested LCOs are able to mark selectively CSCs, however in a case of two molecules: pHTMI and p-HTAA we observed that a relatively rare population of cells (in few cell lines) are stained more intensively than the bulk of cancer cells. Hypothetically, this subset of cells may represent cancer stem cell population, although certainly further experiments are necessary to back that hypothesis. The hypothesis is supported mainly by the previous data obtained on the aforementioned LCOs. Thus one cannot exclude that especially in breast cancer lines SKBR3 and MDAMB-468, and MCF-7, that are known for the presence of CSC, p-HTMI or p-HTAA are able to mark stem cells. Further experiments with co-labeling with specific markers of CSCs for each cell line and cell sorting will be carried out in the future.

Recent data on co-localization of mutant p53 and amyloid-like protein aggregates presented by J. L Silva, and coworkers $(23,24)$, together with fact that LCOs can mark amyloid structures prompted us to explore whether the lack of functional p53 in cells can alter the cell 
stainability by our newly developed LCOs. As p-HTAA is proven to be an amyloid ligand (5), we suggest that the decreased staining of HCT $\mathrm{p} 53^{-/-}$observed in our studies might be due to the presence of less aggregated p53 in cells. The situation seems to be more intriguing when it comes to staining by p-HTMI. We suspect that this LCO, as postulated previously, may have molecular targets in cells with stem-cell-like properties. The loss of p53 is more frequently associated with the stem cell-like cellular plasticity that in turn is suggested to drive tumor progression (25-27). Observations that p53 suppresses the self-renewal of stem cells are common in various biological systems $(28,29)$, but despite growing number of evidence linking p53 loss to stem-like phenotypes in cancer, the mechanism of p53 contribution to acquisition of stemness is still not fully understood (26). If p-HTMI is indeed able to interact with biological structures involved in cell stemness, we believe that it may serve as an excellent marker of CSC in the future.

In conclusion, LCO's properties presented in this study make these dyes an alternative to conventional dyes and attractive candidates for further studies. We have shown that newly developed fluorescent conjugated oligothiophenes bind to cellular targets to different extent and thus are able to discriminate various types of cells, especially differing in p53 status. Since some genetic changes may have a profound effect on cell biology (30,31), LCO-derivates may be good candidates to detect specific genetic changes, based on cellular phenotype. Despite the present poor understanding of the mechanisms of phenomena studied here, LCOs should be considered as an important area of research. Investigations to determine molecular targets of LCOs are ongoing.

\section{ACKNOWLEDGMENTS}

Our work is supported by the Swedish Foundation for Strategic Research (K.P.R.N, M.B, K.M). K.P.R.N is financed by an ERC Starting Independent Researcher Grant (Project: MUMID) from the European Research Council. MJL, AC-P and MVJ kindly acknowledge the core/startup support from Linkoping University, from Integrative Regenerative Medicine Center (IGEN), from Cancerfonden (2013/391), and from VR-NanoVision (K2012-99X -22325-01-5). 


\section{LITERATURE}

1. Barbarella G, Melucci M, Sotgiu G. The versatile thiophene: An overview of recent research on thiophene-based materials. Advanced Materials 2005;17:1581-1593.

2. Palama I, Di Maria F, Viola I, Fabiano E, Gigli G, Bettini C, Barbarella G. Live-cellpermeant thiophene fluorophores and cell-mediated formation of fluorescent fibrils. J Am Chem Soc 2011;133:17777-85.

3. Capobianco ML, Naldi M, Zambianchi M, Barbarella G. Oligothiophene phosphoramidites for oligonucleotide labelling. Tetrahedron Letters 2005;46:8181-8184.

4. Capobianco ML, Barbarella G, Manetto A. Oligothiophenes as Fluorescent Markers for Biological Applications. Molecules 2012;17:910-933.

5. Aslund A, Sigurdson CJ, Klingstedt T, Grathwohl S, Bolmont T, Dickstein DL, Glimsdal E, Prokop S, Lindgren M, Konradsson P and others. Novel pentameric thiophene derivatives for in vitro and in vivo optical imaging of a plethora of protein aggregates in cerebral amyloidoses. ACS Chem Biol 2009;4:673-84.

6. Sigurdson CJ, Nilsson KP, Hornemann S, Heikenwalder M, Manco G, Schwarz P, Ott $D$, Rulicke T, Liberski PP, Julius $C$ and others. De novo generation of a transmissible spongiform encephalopathy by mouse transgenesis. Proc Natl Acad Sci U S A 2009;106:304-9.

7. Nilsson KP, Joshi-Barr S, Winson O, Sigurdson CJ. Prion strain interactions are highly selective. J Neurosci 2010;30:12094-102.

8. Hammarstrom P, Simon R, Nystrom S, Konradsson P, Aslund A, Nilsson KPR. A Fluorescent Pentameric Thiophene Derivative Detects in Vitro-Formed Prefibrillar Protein Aggregates. Biochemistry 2010;49:6838-6845.

9. Bjork P, Peter RNK, Lenner L, Kagedal B, Persson B, Inganas O, Jonasson J. Conjugated polythiophene probes target lysosome-related acidic vacuoles in cultured primary cells. Mol Cell Probes 2007;21:329-37.

10. Mahajan V, Klingstedt T, Simon R, Nilsson KP, Thueringer A, Kashofer K, Haybaeck J, Denk H, Abuja PM, Zatloukal K. Cross beta-sheet conformation of keratin 8 is a specific feature of Mallory-Denk bodies compared with other hepatocyte inclusions. Gastroenterology 2011;141:1080-1090 e1-7.

11. Nilsson KP, Rydberg J, Baltzer L, Inganas O. Self-assembly of synthetic peptides control conformation and optical properties of a zwitterionic polythiophene derivative. Proc Natl Acad Sci U S A 2003;100:10170-4.

12. Klingstedt T, Aslund A, Simon RA, Johansson LB, Mason JJ, Nystrom S, Hammarstrom P, Nilsson KP. Synthesis of a library of oligothiophenes and their utilization as fluorescent ligands for spectral assignment of protein aggregates. Org Biomol Chem 2011;9:8356-70. 
13. Klingstedt T, Blechschmidt C, Nogalska A, Prokop S, Haggqvist B, Danielsson O, Engel WK, Askanas V, Heppner FL, Nilsson KP. Luminescent conjugated oligothiophenes for sensitive fluorescent assignment of protein inclusion bodies. Chembiochem 2013;14:607-16.

14. Sigurdson CJ, Nilsson KP, Hornemann S, Manco G, Polymenidou M, Schwarz P, Leclerc M, Hammarstrom P, Wuthrich K, Aguzzi A. Prion strain discrimination using luminescent conjugated polymers. Nat Methods 2007;4:1023-30.

15. Margalith I, Suter C, Ballmer B, Schwarz P, Tiberi C, Sonati T, Falsig J, Nystrom S, Hammarstrom P, Aslund A and others. Polythiophenes inhibit prion propagation by stabilizing prion protein (PrP) aggregates. Journal of Biological Chemistry 2012;287:18872-87.

16. Chang HY, Chi JT, Dudoit S, Bondre C, van de Rijn M, Botstein D, Brown PO. Diversity, topographic differentiation, and positional memory in human fibroblasts. Proc Natl Acad Sci U S A 2002;99:12877-82.

17. Goodpaster T, Legesse-Miller A, Hameed MR, Aisner SC, Randolph-Habecker J, Coller HA. An immunohistochemical method for identifying fibroblasts in formalinfixed, paraffin-embedded tissue. J Histochem Cytochem 2008;56:347-58.

18. Reya T, Morrison SJ, Clarke MF, Weissman IL. Stem cells, cancer, and cancer stem cells. Nature 2001;414:105-11.

19. Farahani E, Patra HK, Jangamreddy JR, Rashedi I, Kawalec M, Rao RK, Batakis P, Wiechec E. Cell adhesion molecules and their relation to (cancer) cell stemness. Carcinogenesis 2014;35.

20. Hombach-Klonisch S, Panigrahi S, Rashedi I, Seifert A, Alberti E, Pocar P, Kurpisz M, Schulze-Osthoff K, Mackiewicz A, Los M. Adult stem cells and their transdifferentiation potential--perspectives and therapeutic applications. J Mol Med (Berl) 2008;86:1301-14.

21. Hombach-Klonisch S, Paranjothy T, Wiechec E, Pocar P, Mustafa T, Seifert A, Zahl C, Gerlach KL, Biermann K, Steger K and others. Cancer stem cells as targets for cancer therapy: selected cancers as examples. Arch Immunol Ther Exp (Warsz) 2008;56:165-80.

22. Wasik AM, Grabarek J, Pantovic A, Cieślar-Pobuda A, Asgari HR, Bundgaard-Nielsen C, Rafat M, Dixon IMC, Ghavami S, Łos MJ. Reprogramming and carcinogenesis parallels and distinctions. Int. Rev. Cell Mol. Biol. 2014;308.

23. Bom APDA, Rangel LP, Costa DCF, de Oliveira GAP, Sanches D, Braga CA, Gava LM, Ramos CHI, Cepeda AOT, Stumbo AC and others. Mutant p53 Aggregates into Prionlike Amyloid Oligomers and Fibrils IMPLICATIONS FOR CANCER. Journal of Biological Chemistry 2012;287:28152-28162.

24. Levy CB, Stumbo AC, Bom APDA, Portari EA, Cordeiro Y, Silva JL, DeMoura-Gallo CV. Co-localization of mutant p53 and amyloid-like protein aggregates in breast tumors (vol 43, pg 60, 2011). International Journal of Biochemistry \& Cell Biology 2011;43:946-946. 
25. Jerry DJ, Tao LW, Yan HH. Regulation of cancer stem cells by p53. Breast Cancer Research 2008;10.

26. Spike BT, Wahl GM. p53, Stem Cells, and Reprogramming: Tumor Suppression beyond Guarding the Genome. Genes Cancer 2011;2:404-19.

27. Godar S, Ince TA, Bell GW, Feldser D, Donaher JL, Bergh J, Liu A, Miu K, Watnick RS, Reinhardt F and others. Growth-inhibitory and tumor-suppressive functions of p53 depend on its repression of CD44 expression. Cell 2008;134:62-73.

28. Zhang M, Behbod F, Atkinson RL, Landis MD, Kittrell F, Edwards D, Medina D, Tsimelzon A, Hilsenbeck S, Green JE and others. Identification of tumor-initiating cells in a p53-null mouse model of breast cancer. Cancer Research 2008;68:46744682.

29. Meletis K, Wirta V, Hede SM, Nister M, Lundeberg J, Frisen J. P53 suppresses the self-renewal of adult neural stem cells. Development 2006;133:363-369.

30. Ghavami S, Hashemi M, Ande SR, Yeganeh B, Xiao W, Eshraghi M, Bus CJ, Kadkhoda $\mathrm{K}$, Wiechec E, Halayko AJ and others. Apoptosis and cancer: mutations within caspase genes. J Med Genet 2009;46:497-510.

31. Wiechec E. Implications of genomic instability in the diagnosis and treatment of breast cancer. Expert Rev Mol Diagn 2011;11:445-53. 